\title{
Impact of Cross-axis Structural Dynamics on Validation of Linear Models for Space Launch System
}

\author{
Jing Pei $^{1}$, Stephen D. Derry ${ }^{2}$, Zhiqiang Zhou ${ }^{3}$ \\ NASA Langley Research Center, Hampton VA 23681 \\ Jerry R. Newsom ${ }^{4}$ \\ Analytical Mechanics Associates, Hampton VA 23681
}

\begin{abstract}
A feasibility study was performed to examine the advisability of incorporating a set of Programmed Test Inputs (PTIs) during the Space Launch System (SLS) vehicle flight. The intent of these inputs is to provide validation to the preflight models for control system stability margins, aerodynamics, and structural dynamics. During October 2009, Ares I-X program was successful in carrying out a series of PTI maneuvers which provided a significant amount of valuable data for post-flight analysis. The resulting data comparisons showed excellent agreement with the preflight linear models across the frequency spectrum of interest. However unlike Ares I-X, the structural dynamics associated with the SLS boost phase configuration are far more complex and highly coupled in all three axes. This presents a challenge when implementing this similar system identification technique to SLS. Preliminary simulation results show noticeable mismatches between PTI validation and analytical linear models in the frequency range of the structural dynamics. An alternate approach was examined which demonstrates the potential for better overall characterization of the system frequency response as well as robustness of the control design.
\end{abstract}

\section{Introduction}

System identification maneuvers have frequently been used as part of aircraft flight test programs ${ }^{1-4}$. Similar techniques were utilized in the October 2009 test flight of the Ares I-X launch vehicle ${ }^{5-7}$. On Ares I-X, the Programmed Test Inputs (PTIs) were performed during three distinct phases of the two-minute ascent trajectory: transonic, maximum dynamic pressure, and high supersonic Mach prior to Solid Rocket Booster (SRB) burnout. The test inputs were orthogonalized so that the pitch and yaw axes could be excited concurrently. Post-flight results provided engineers with valuable flight data used to validate the preflight models and frequency response. Due to the successful execution of the PTIs on Ares I-X, a study was conducted to assess the feasibility of applying a similar technique to the Space Launch System (SLS).

In the formulation of launch vehicle flight dynamics, the standard practice is to use a set of linear-orthogonal slosh and elastic models in combination with nonlinear rigid body dynamics. For the purpose of designing a control system, the plant is linearized at various times throughout the trajectory and assumes decoupling in the roll, pitch, and yaw channels. Single-Input Single-Output (SISO) classical stability analysis such as Bode, Nichols, and Nyquist diagrams are used to evaluate the gain and phase margins associated with the rigid body, slosh, and elastic modes (free-free) of the system. The Ares I-X configuration was axisymmetric which consisted of a cylindrical dummy upper stage integrated with a Space Shuttle SRB. Due to the lack of cross axis coupling in the system dynamics, the post-flight linear models generated based on PTI telemetry data showed excellent agreement with the preflight SISO linear models ${ }^{5}$. However, the SLS structural dynamics model during boost phase (prior to SRB separation) is far more complex, consisting of a core stack and two strap-on boosters along with four fully actuated RS-25 core stage engines (CSE). Examination of the spatial shape functions or mode shapes of the vehicle Finite

\footnotetext{
${ }^{1}$ Aerospace Engineer, Systems Analysis and Concepts Directorate, Vehicle Analysis Branch

${ }^{2}$ Aerospace Engineer, Research Directorate, Dynamic Systems and Control Branch

${ }^{3}$ Aerospace Engineer, Research Directorate, Dynamic Systems and Control Branch

${ }^{4}$ Aerospace Engineer, Research Directorate, Dynamic Systems and Control Branch
} 
Element Models (FEM) indicates that many modes appear to be global core bending modes in one axis also exhibit considerably large mode shapes of the SRBs in another axis. This multi-axis characteristic is likely due to asymmetry in the vehicle mass and inertia as well as differences in the interconnect (core stage and SRBs) stiffnesses amongst the various degrees of freedom. Such behavior is persistent for many modes throughout the boost flight segment. When the slightly modified Ares I-X PTIs were applied to the SLS system, simulation results showed a noticeable mismatch between the analytical SISO linear models and the generated frequency response based on control system data.

The overall purpose of this paper is to illustrate potentially how multi-axis characteristics of the structural dynamics would affect the system identification results. This paper is organized as follows: Section 2 provides an overview of the PTI maneuver feasibility study and analysis. Section 3 provides a description of the multi-axis behavior of the SLS flex modes during the boost phase. A modal gain criteria was developed to evaluate the degree of cross axis coupling associated with a particular mode. Section 4 illustrates how the multi-axis structural modes affect the system identification results with comparisons between the simulated experimental and analytical linear models are presented. Section 5 concludes the discussion and summarizes the findings.

\section{PTI Background and Overview}

PTI waveforms similar to those used on Ares I-X were implemented in this feasibility study. The PTIs are continuous sets of angular acceleration commands injected into the system downstream of the Flight Control System (FCS) commands and prior to the allocator shown in Fig. 1. The overall design philosophy behind the PTI maneuvers is to have as little impact on the trajectory as possible while maximizing the amount of data collected. The SLS ascent trajectory to low earth orbit consists of two distinct phases: boost phase (prior to SRB separation) and core phase (post SRB separation). The boost phase is of particular interest due to the high dynamic pressure (when aerodynamic forces are large) and significant cross-axis coupling characteristics associated with certain flex modes.

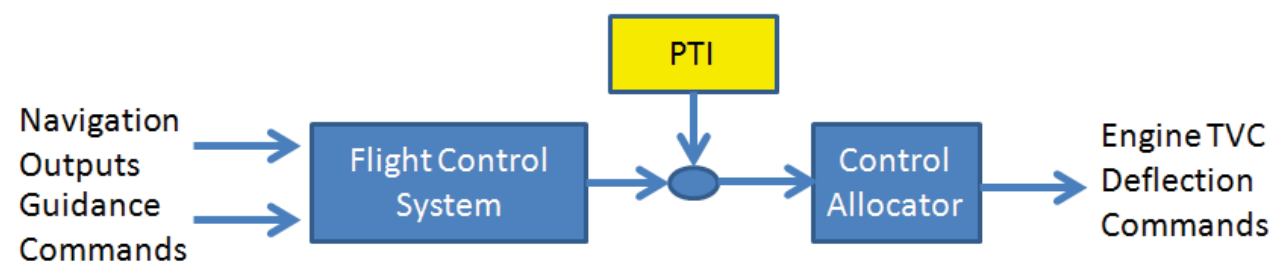

Figure 1. Schematic FCS \& PTI

During the simulation, the PTI commands were scheduled as a sequence of 10 second-long optimized multi-sine Thrust Vector Control (TVC) sweep inputs designed to excite the system dynamics at discrete frequencies up to 2.5 Hz. The pitch signal has content at even frequency points $(0.2,0.4, \ldots 2.4 \mathrm{~Hz})$, while the yaw signal is at odd frequency $(0.3,0.5, \ldots 2.5 \mathrm{~Hz})$ to avoid mutual interference. Multi-sine waveforms were selected because they guarantee persistent excitation across the frequency range of interest ${ }^{1}$. The waveforms start and end at zero and are roughly symmetric about the reference condition. A single 10-second sample of the PTI waveform used for excitation is shown in Fig 2. Note: the axes labels were taken off some figures to meet International Traffic in Arms Regulations (ITAR) requirements. 


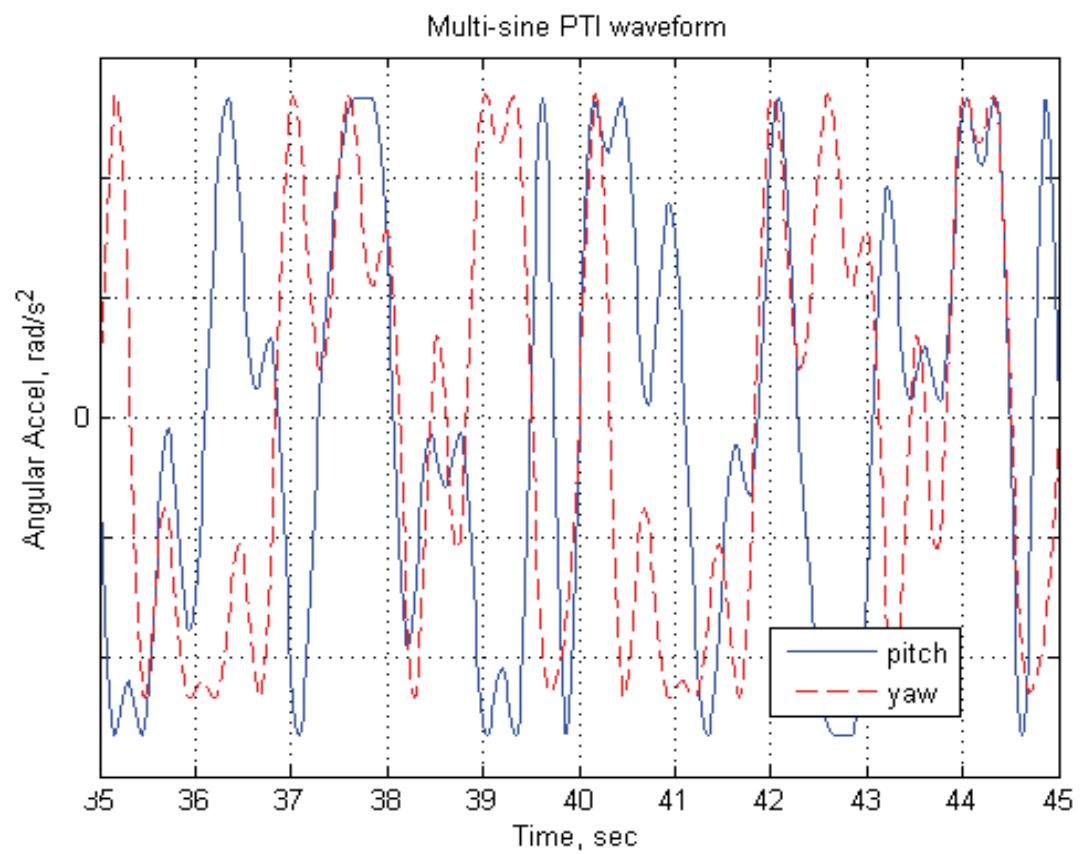

Figure 2. PTI excitation waveform

The associated power distribution for a given multi-sine sweep is shown in Fig 3. The power is biased towards the low frequency spectrum and decreases with increasing frequency to avoid excessive TVC actuator rates. The amplitude of the maneuver is a compromise between keeping the input magnitude low enough to avoid loads issues while high enough to overcome sensor noise and get sufficient vehicle response. Due to the rapidly changing environment and system dynamics, the maneuver time window at a fixed flight condition is extremely limited. To maximize the amount of data acquired, the inputs are designed to simultaneously excite both the pitch and yaw channels. The pitch and yaw waveforms are mutually orthogonal in both time and frequency domains such that each axis can be identified without interference from the other despite being exercised simultaneously ${ }^{5}$.

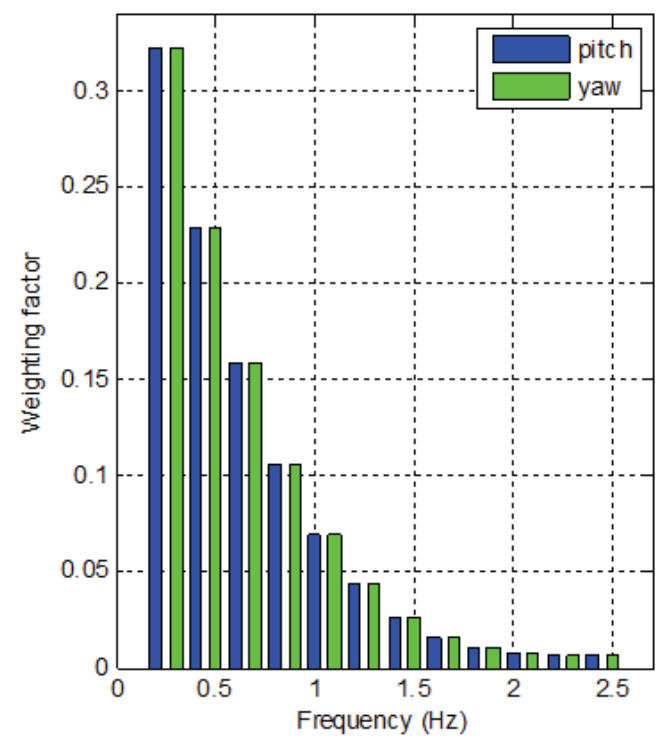

Figure 3. Sweep Power Distribution

American Institute of Aeronautics and Astronautics 
Analysis techniques developed and implemented for Ares I-X were used to determine the frequency domain results from the PTIs in this study. The frequency response method constitutes a non-parametric analysis because it characterizes the input-output behaviors without having to define a model parameter ${ }^{4}$. Parametric models such as transfer function modeling attempt to fit a low-order set of gains, poles, zeros, and time delays that best represent the frequency response data. However, these parameters may lack physical meaning as the closed-loop SLS system consists of several hundred states. A loss of intuitiveness is an inherent problem when representing a high-order system with an equivalent low-order counterpart. Therefore, SLS parametric modeling may not offer additional insight into the plant dynamics compared to the non-parametric approach, hence was not considered.

The system frequency response can be estimated using only control system telemetry. This is done by comparing the command signals going into the allocator (FCS + PTI) with the response (FCS) in the frequency domain. Figure 5 shows the pitch plane command and response from the simulation for a 10 second maneuver interval. The sampled time domain data $(50 \mathrm{~Hz})$ were subsequently converted to the frequency domain using a Chirp z-Transform with cubic interpolation ${ }^{3}$. A 10-second Hann window, coinciding with the 10 second PTI sweep is applied to the command and response data prior to conversion of the data to the frequency domain for improved identification results. Finally, a frequency response function is used to determine the discrete gain and phase of the system.

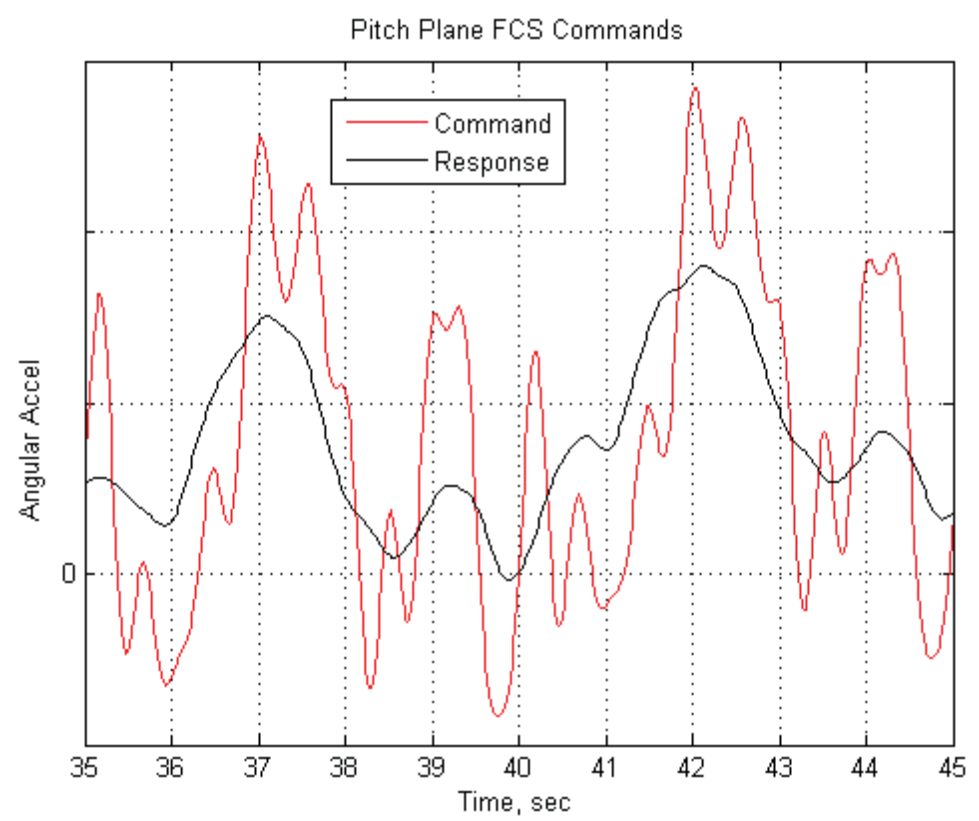

Figure 5. Pitch plane FCS Commands

\section{SLS Structural Dynamics Model}

The structural dynamics model associated with the Ares 1-X boost phase is more complex compared to the SLS core phase due to the presence of the two SRBs. A modal gain metric is used to evaluate the impact that a particular mode has on the overall global bending and the associated degree of cross-axis coupling. The modal gain or residue $^{8}$ of an elastic mode can be computed as the product of the controllability and observability coefficients. The controllability coefficients are the mode shapes, $\varphi$ at the thrust application locations (2 SRB and 4 CSE in this case). The observability coefficients are the mode slopes, $\psi$ at the 3 sensor locations: Inertial Navigation Unit (INU), Forward Rate Gyro Assembly (FRGA), and Aft Rate Gyro Assembly (ARGA). Figure 6 shows the approximate locations of the Thrust Application points (TA) and the sensors. 


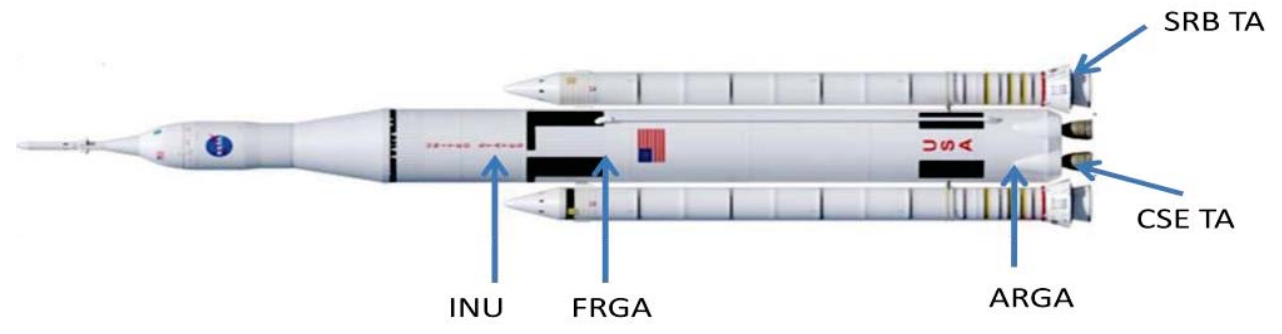

Figure 6. Thrust and Sensor Locations

The modal gain is a 2x2 matrix relating the controllability and observability of the pitch and yaw axes for each input-to-output pair. Equation 1 is a representation of the modal gain formulation between the $i^{\text {th }}$ engine and $j^{\text {th }}$ sensor. The roll axis is ignored due to the lack of coupling with the others two axes during the boost phase. The 1-1 term in the matrix represents the product of pitch mode shape at the engine with the pitch mode slope at the sensor. The 1-2 term consists of the pitch mode shape at engine with the yaw mode slope at the sensor and so forth. A large value in the diagonal elements indicates either a dominate pitch or yaw mode, whereas large off diagonal elements indicate strong cross axis coupling.

$$
\mathrm{MG}_{\mathrm{ij}}=\left[\begin{array}{ll}
\varphi, \mathrm{P}, \mathrm{i} \psi, \mathrm{P}, \mathrm{j} & \varphi, \mathrm{P}, \mathrm{i} \psi, \mathrm{Y}, \mathrm{j} \\
\varphi, \mathrm{Y}, \mathrm{i} \psi, \mathrm{P}, \mathrm{j} & \varphi, \mathrm{Y}, \mathrm{i} \psi, \mathrm{Y}, \mathrm{j}
\end{array}\right]
$$

Figure 7 is a plot of all four elements of the modal gain matrix for each engine-sensor pair for the 8 lowest frequency modes from the FEM associated with 35 to 45 seconds in the simulation. Note: all results shown in this paper are for this FEM model unless stated otherwise. The $\mathrm{x}$-axis represents all the engine-sensor combinations (18 total). Modes 1 and 2 are the first yaw and pitch bending modes. Mode 3, 6, and 7 are higher frequency bending modes with significant pitch input - yaw output coupling characteristics exhibited by certain engine-to-sensor pairs.
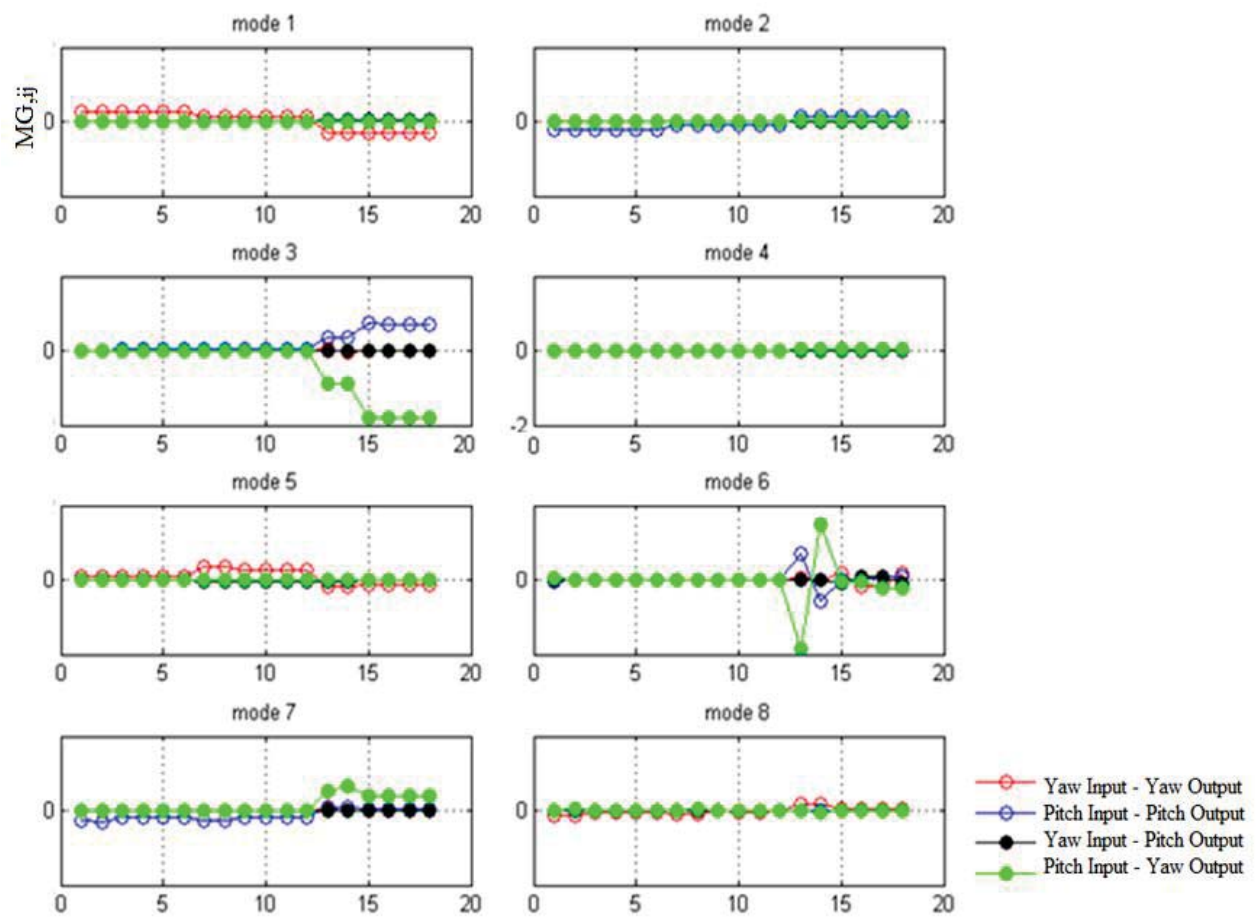

Figure 7. Modal Gain Matrix for Modes 1-8 
Figures 8a and 8b are plots of the pitch and yaw mode shapes and slopes associated with mode 3. The mode shapes and slopes at the sensor and engine locations are marked in cyan and blue/pink respectively. The large pitch mode shapes at the engine gimbals in conjunction with the large ARGA yaw mode slopes indicates strong pitch-yaw coupling. This observation is consistent with the large pitch input - yaw output component of the modal gain matrix (green circles) associated with mode 3 shown in Fig 7.
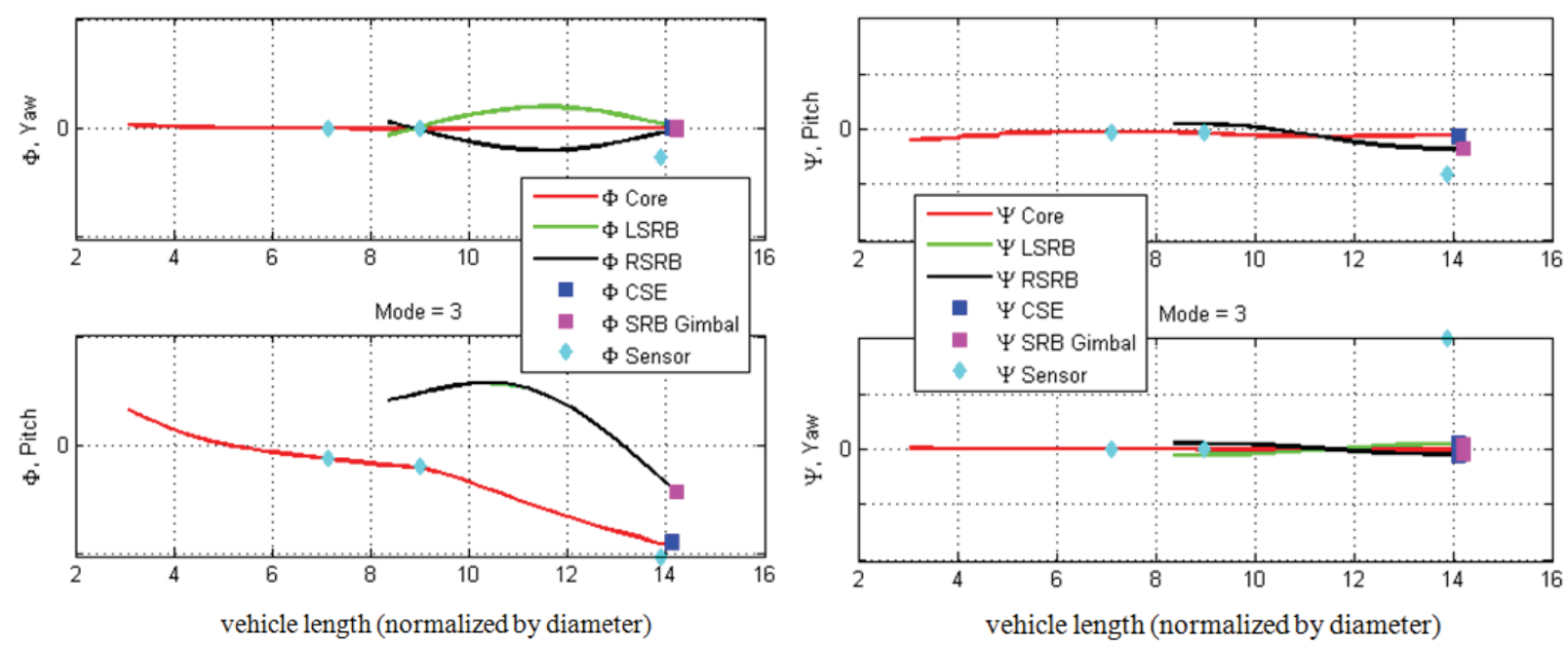

Figure 8a, 8b. Mode Shapes and Slopes: Mode 3

An overall modal gain indicator was developed to assess the contribution of the $\mathrm{k}^{\text {th }}$ flex mode on the pitch/yaw rate sensed by the FCS. This is computed as the product of the $\mathrm{j}^{\text {th }}$ pitch/yaw blending gains $\left(\alpha_{\mathrm{j}}\right)$ with each element of the modal gain matrix (Eq. 2) associated with pitch (1-1 and 2-1 terms) or yaw (1-2 and 2-2 terms) output. These individual products are then summed over all engine $(n)$ and sensor $(m)$ combinations. Equation 2 provides the analytical expression of the overall pitch plane modal gain indicator for the $\mathrm{k}^{\text {th }}$ flex mode. A similar approach is taken for the yaw plane.

$$
\begin{gathered}
\operatorname{MGI}_{\mathrm{P}, \mathrm{k}}=\left[\sum_{j=1}^{m} \sum_{i=1}^{n} \alpha_{\mathrm{P}, \mathrm{j}} \varphi_{\mathrm{P}, \mathrm{i}} \psi_{\mathrm{P}, \mathrm{j}} \sum_{j=1}^{m} \sum_{i=1}^{n} \alpha_{\mathrm{P}, \mathrm{j}} \varphi_{\mathrm{Y}, \mathrm{i}} \psi_{\mathrm{P}, \mathrm{j}}\right] \\
\sum_{j=1}^{m} \alpha_{\mathrm{P}, \mathrm{j}}=1
\end{gathered}
$$

Figure 9 is a graphical result of the overall pitch and yaw modal gain indicators expressed in Eq. 2 for all modes below $3 \mathrm{~Hz}$. Subplot 1 provides an indication on the contribution of the $\mathrm{k}^{\text {th }}$ mode on pitch rate sensed by the FCS due to either a pitch or yaw input. Yaw plane results are provided in subplot 2. A black marker with large magnitude indicates that the $\mathrm{k}^{\text {th }}$ mode is predominating either in pitch or yaw. This directly corresponds to peaks in the Bode magnitude plots shown in Fig 10. The Bode plots correspond to the diagonal elements in the open loop transfer function matrix which do not capture any cross-axis effects; hence they do not capture the pitch-yaw, yawpitch components of the modal gain indicator. Section IV illustrates the impact of axis coupling associated with a particular flex mode on the validation linear models generated based on the PTI maneuvers. 

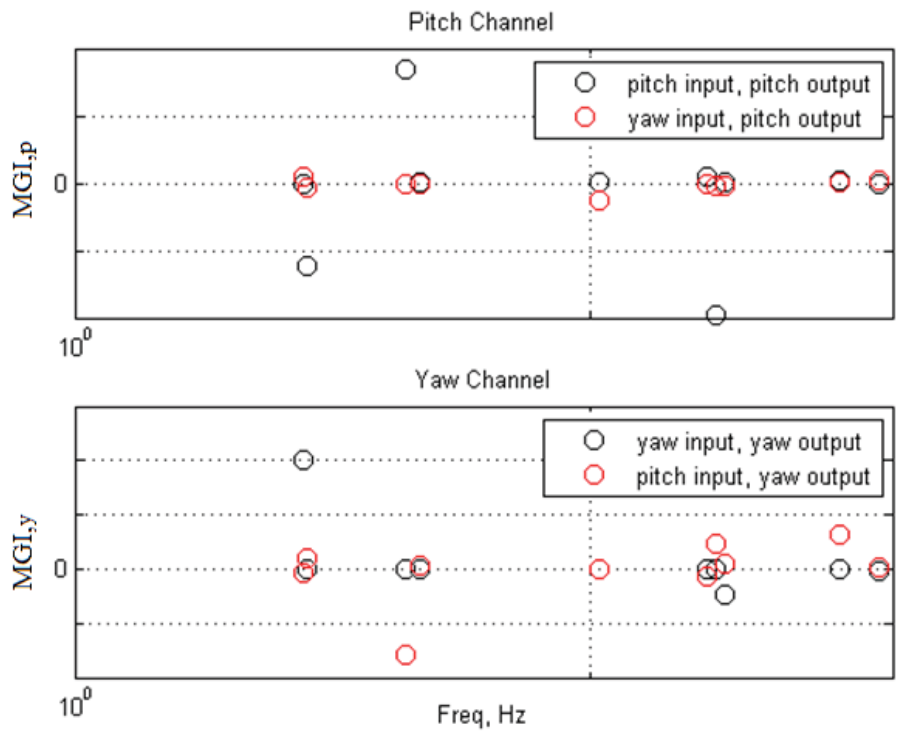

Figure 9. Overall Modal Gain Indicator, Pitch and Yaw

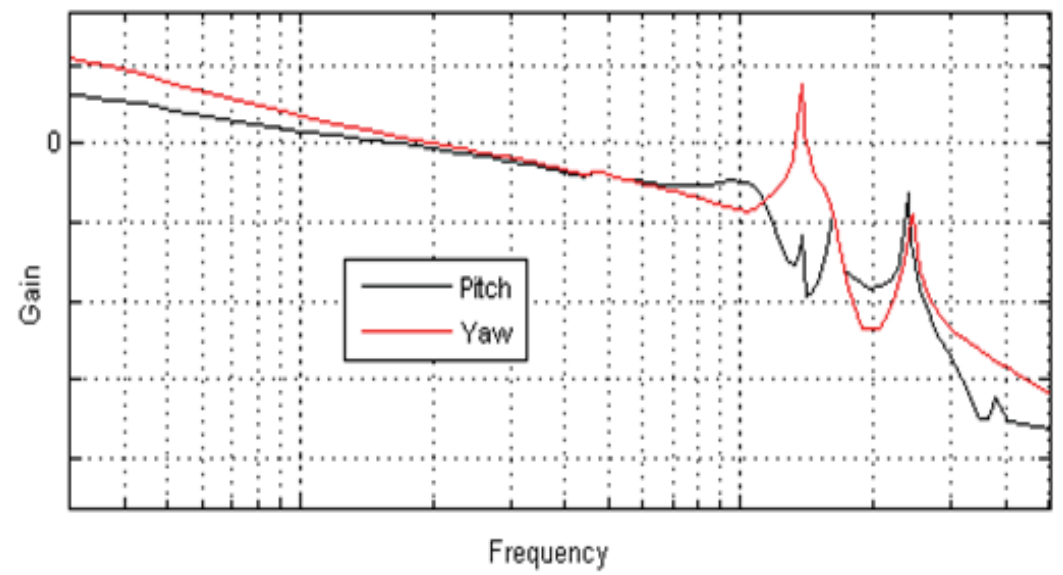

Figure 10. Analytical Pitch and Yaw SISO Bode Magnitude Plots

\section{Simulation Results}

The Space Transportation and Aeronautics Research Simulation (STARS) developed at NASA Langley Research Center was used to generate the analytical and simulated experimental linear models. STARS is MATLAB/Simulink ${ }^{\circledR}{ }^{9}$ based and uses numerical perturbation to compute linear models at any given time in the trajectory. Figure 11 shows the pitch Bode gain plot corresponding to $\mathrm{T}=40$ seconds. The discrete frequency responses based on the PTI results (maneuver window between 35 and 45 seconds) are co-plotted. Figure 11 depicts good agreement between the analytical and simulated experimental models. 


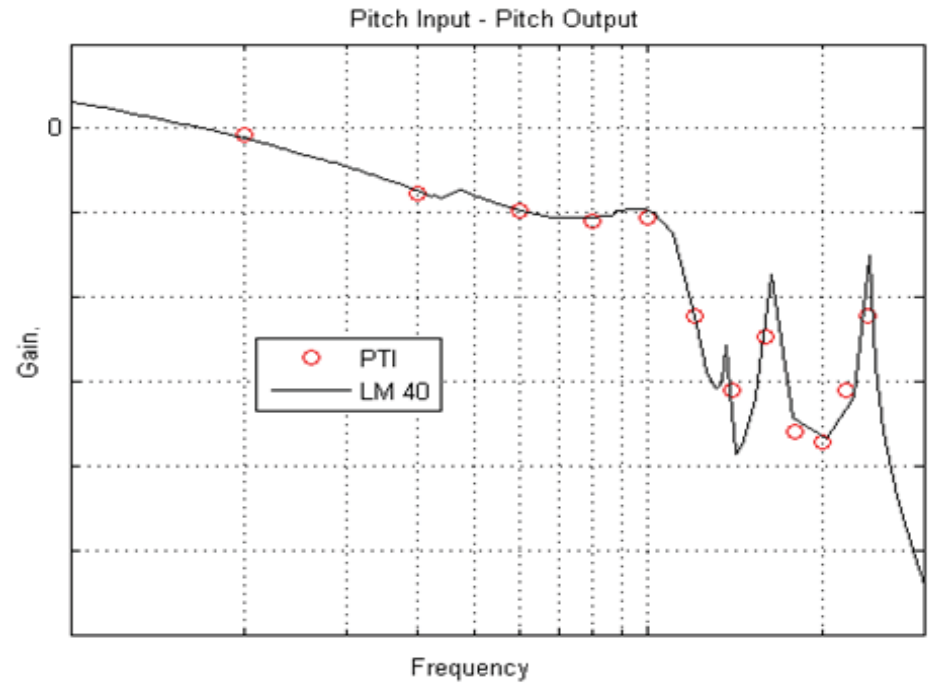

Figure 11. P-P Bode Magnitude Plot, Analytical vs. Simulated Experimental

Figures 12 depicts the yaw response during the same 10 second interval. The PTI results match the analytical linear model quite well at the lower frequencies; however, a poor agreement is exhibited at the flex frequencies. The strong pitch-yaw coupling characteristic exhibited by modes 3 and 7 is responsible for the mismatches. For instance, it is apparent the yaw magnitude Bode associated with the analytical linear model is much greater compared to the highest-frequency PTI frequency response. Modes 7 and 8 are closely spaced in frequency; therefore they both contribute to the estimated frequency response at the high frequency end. Essentially the significant pitch-yaw coupling exhibited by mode 7 is canceling out the yaw-yaw peak of mode 8.

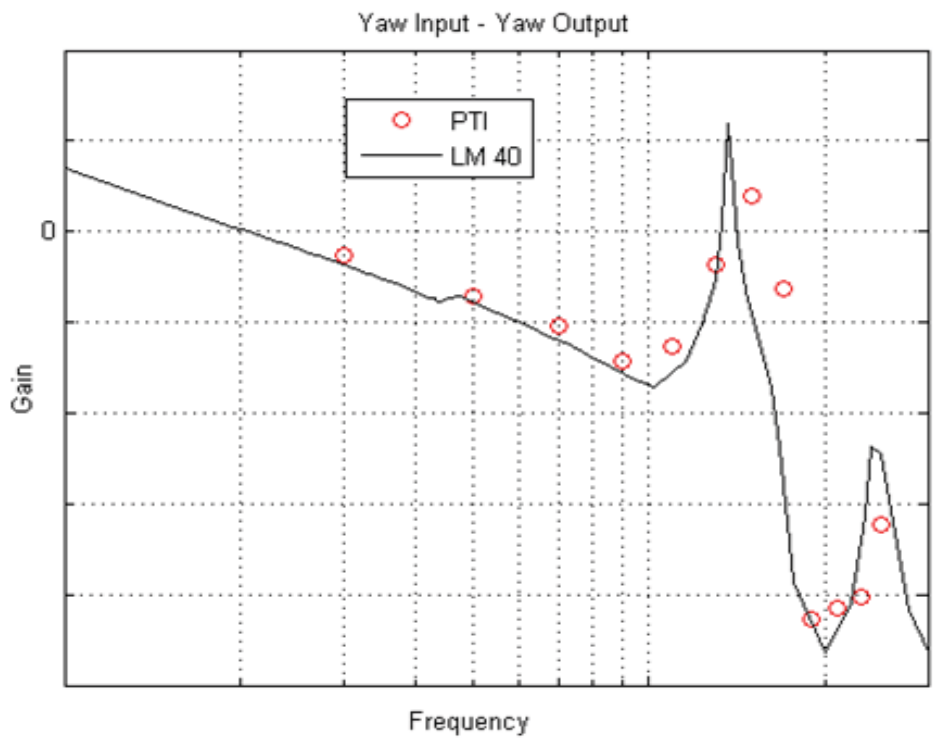

Figure 12. Y-Y Bode Magnitude Plot, Analytical vs. Simulated Experimental

This mismatch in the yaw frequency response at higher frequencies is persistent throughout the SLS boost phase due to the inherent flex pitch-yaw coupling. The coupling characteristics in the structural dynamics model is not observed during the core phase of flight after the SRBs are released. Hence, the frequency response validation agrees with the analytical linear models in both pitch and yaw for those selected time intervals. The core stage flight results are not shown in this paper. Results from this study suggest that the off-diagonal terms in the open loop 
transfer function matrix (OLTFM) for SLS boost phase are more significant compared to Ares I-X due to asymmetry in the vehicle configuration.

$$
\left[\begin{array}{ll}
\frac{Y_{p}}{U_{p}} & \frac{Y_{P}}{U_{y}} \\
\frac{Y_{y}}{U_{p}} & \frac{Y_{y}}{U_{y}}
\end{array}\right]=\left[\begin{array}{ll}
\frac{G_{p p} U_{p}+G_{p y} U_{y}}{U_{p}} & \frac{G_{p p} U_{p}+G_{p y} U_{y}}{U_{y}} \\
\frac{G_{y y} U_{y}+G_{y p} U_{p}}{U_{p}} & \frac{G_{y y} U_{y}+G_{y p} U_{p}}{U_{y}}
\end{array}\right]
$$

Equation 3 illustrates the system to be determined from the PTI analysis. The pitch output $Y_{p}$ from the PTI experiment consists of contributions from both the pitch-pitch and pitch-yaw dynamics. It is apparent that if the pitch-yaw term magnitude is insignificant, then the PTI generated frequency response would agree with the analytical linear model as with the pitch channel in Figure 11. The yaw-pitch dynamics is more significant hence the mismatch in the yaw channel. It is also apparent that the off diagonal terms would not be able to be identified when both channels are excited simultaneously due to coupling of the output signals. For instance, the identification of $G_{p y}$ would be corrupted by the contribution from $G_{p p} U_{p}$.

An alternative approach would be to excite the system with the PTI one axis at a time. It is apparent from Eq. 3 that this would lead to better identification of both the diagonal and off-diagonal transfer functions as the magnitude of $U_{p}$ or $U_{y}$ would be reduced. Neither of the inputs could ever be completely eliminated due to the feedback signals from the FCS needed to stabilize the vehicle. Figure 13 shows the pitch-yaw identification results when PTI is active in both pitch and yaw versus when active only in pitch. The simultaneously excitation approach fails to characterize the $\mathrm{G}_{\mathrm{yp}}$ transfer function as expected. Figures 14a and 14b show the yaw-yaw identification results. Significant improvement in matching can be seen at the higher frequencies with the single axis approach. The obvious drawback is that flight data would be available for only one axis at a particular flight condition.

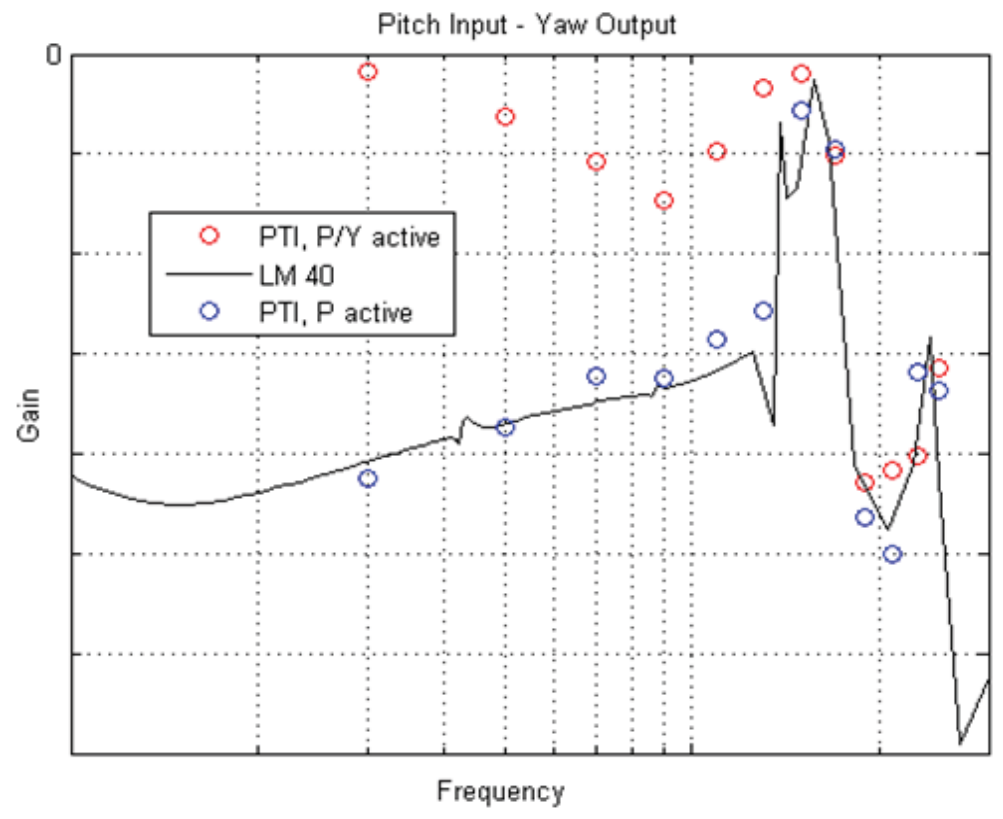

Figure 13. Y-P Magnitude Bode, Single Axis vs. Simultaneous Excitation 


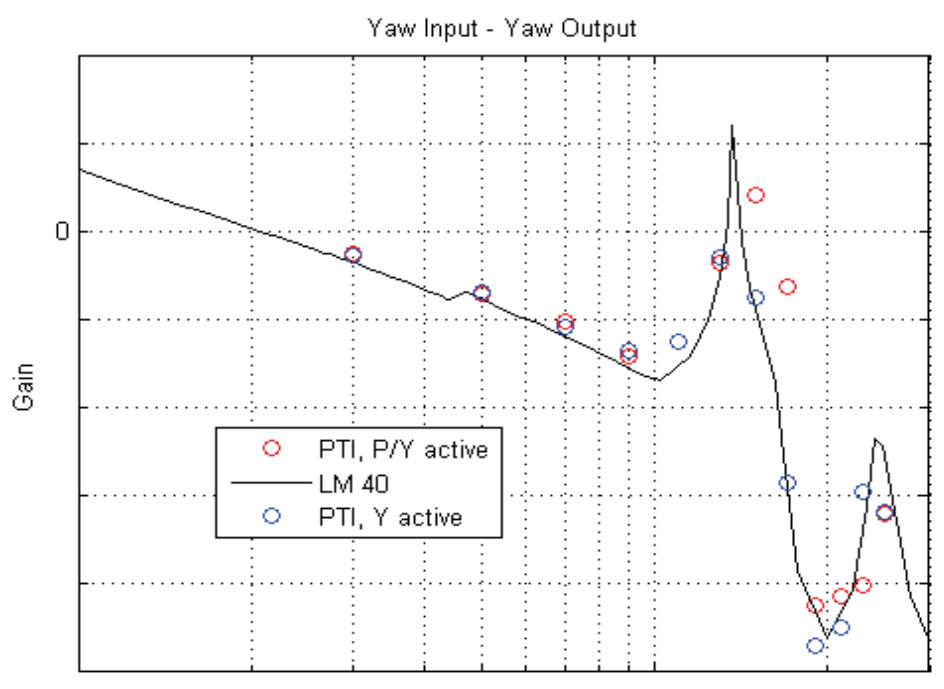

Frequency

Figure 14a. Y-Y Magnitude Bode, Single Axis vs. Simultaneous Excitation

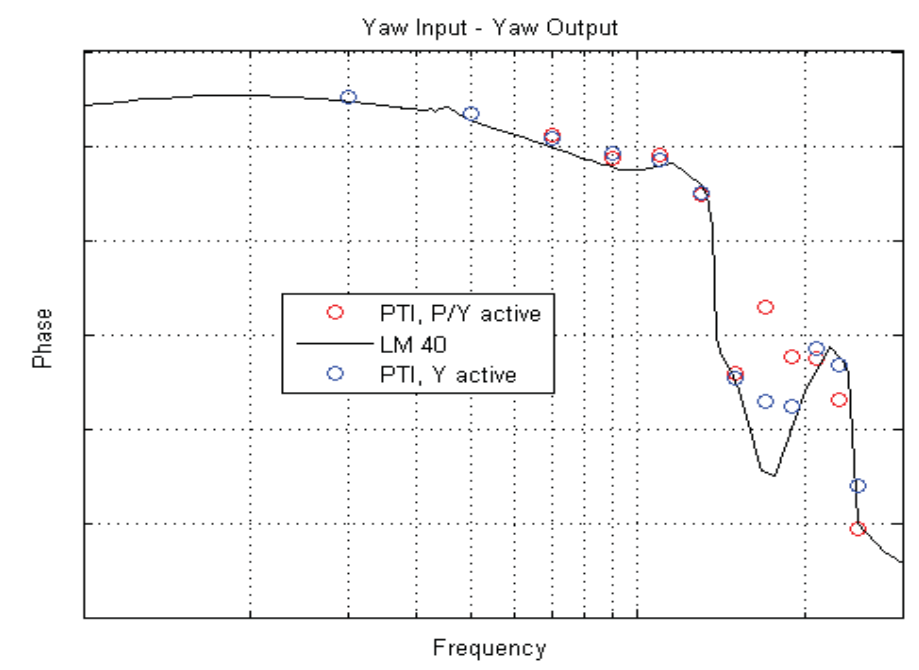

Figure 14b. Y-Y Phase Bode, Single Axis vs. Simultaneous Excitation

The shortcomings of the single axis approach can be mitigated by reducing the excitation time while excecuting the PTI maneuver between the two axes in an alternating fashion. The underlying assumption of this approach is that the vehicle dynamics do not vary much between consecutive maneuvers. A frequency response matrix (2x2) can be then constructed and compared with the analytical transfer function matrix centered at a given PTI cycle (pitch followed by yaw or vice versa). Instead of analyzing each term in the transfer function matrix individually, another way would be to compare the singular values $(\Sigma)$ of the system. For a coupled system, MIMO analysis methods ${ }^{10,11}$ should be used in conjunction with the classical SISO methods even though it is not standard practice for launch vehicle programs to state stability requirements in terms of MIMO metrics. Due to the multi-axis characteristics exhibited by the SLS structural dynamics, singular values provide additional insights into the behavior of the system. For instance, the minimum singular values associated with the return difference matrix can be used to evaluate the stability robustness of a multivariable system ${ }^{12}$. Equation 4 can be used to perform the singular value decomposition, $U$ and $V$ are the output and input directions associated with minimum and maximum singular values ${ }^{11}$.

$$
\mathrm{M}=U \Sigma V^{*}
$$

10

American Institute of Aeronautics and Astronautics 
Figure 15 is a multivariable Bode plot comparing the singular values of the analytical linear model (roll channel not included) with the simulated experimental frequency response generated using the single axis maneuver approach. The plot suggests that coupling is insignificant at the lower frequencies where discrepancies between the maximum and minimum singular values are minimal. At higher frequencies the discrepancies become more pronounced which is consistent with the structural dynamics of the vehicle and the bending filter design. The single axis approach shows it should be able to better characterize the diagonal and off diagonal terms in the transfer function matrix compared to simultaneous excitation.

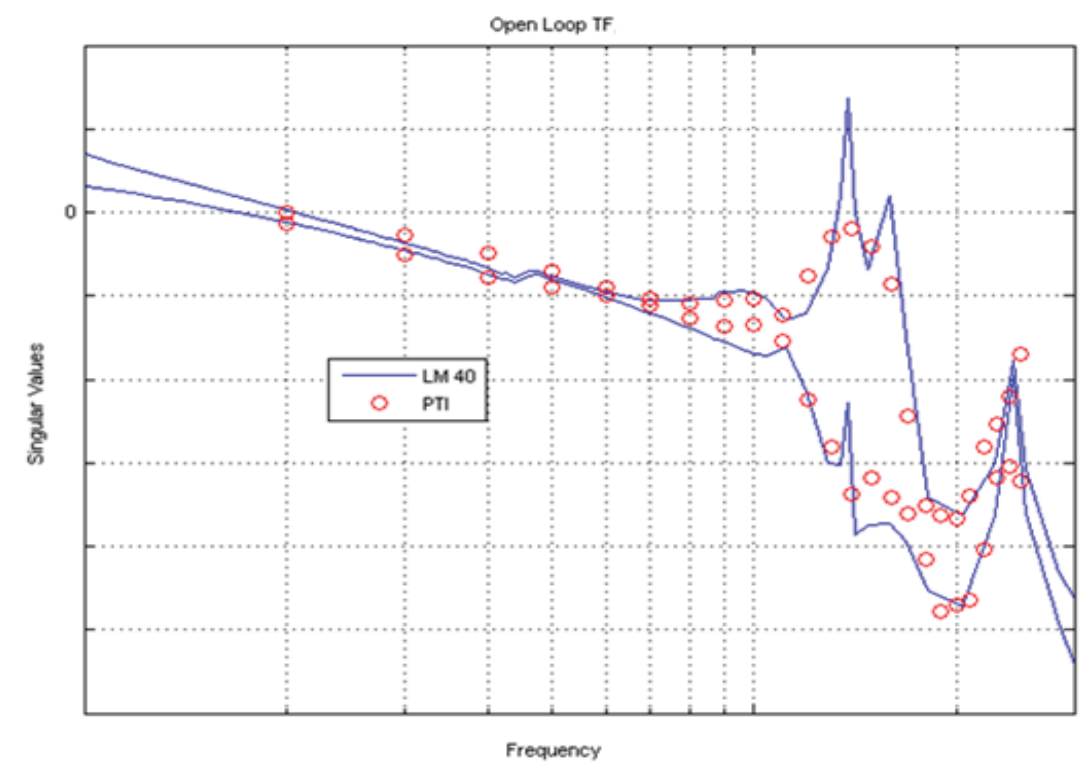

Figure 15. Multivariable Bode Plot, Analytical vs. Simulated Experimental (Single Axis Excitation)

\section{Conclusion}

This paper highlights the cross axis coupling in the plant model and its effect on the system identification results for the SLS vehicle during the boost phase. Waveforms and analysis techniques similar to those implemented for Ares I-X were used in this feasibility study. Due to its asymmetric configuration, the SLS structural dynamics during the boost phase are more complex and exhibit greater cross-axis coupling than was exhibited for the Ares I-X flight test. A modal gain criteria was developed to quantify the degree of coupling. As a result, simulation results indicated that the simulated experimental frequency response from the PTI did not match the analytical linear models at the flex frequencies given the simultaneous excitation approach used during Ares I-X. An alternate PTI technique was demonstrated in which the control maneuvers were performed one axis at a time. Preliminary results indicated that the single axis method provided better identification of the elements in the open loop transfer function matrix and enables verification of the singular values of the system. However, limiting the PTI maneuvers to one axis at a time reduces the quantity of data that can be acquired at a particular flight condition.

\section{References}

${ }^{1}$ Morelli, E. A. "Multiple Input Design for Real-Time Parameter Estimation in the Frequency Domain," Paper REG-360, 13th IFAC Conference on System Identification. August 2003, Rotterdam.

${ }^{2}$ Morelli, E. A. "High Accuracy Evaluation of the Finite Fourier Transform Using Sampled Data,” NASA TM 110340. June 1997.

${ }^{3}$ Klein, V., Morelli, E. A. Aircraft System Identification. AIAA, Reston VA, 2006. 
${ }^{4}$ Tischler, M. Remple, R. Aircraft and Rotorcraft System Identification. AIAA, Reston VA, 2006.

${ }^{5}$ Derry, S. “Ares I-X Flight Test System Identification Results,” JANNAF-1844, 58th JANNAF Propulsion Meeting, April 2011.

${ }^{6}$ Brandon, J., Derry, S., Heim, E., Hueschen, R., Bacon, B., “Ares I-X Stability and Control Flight Test: Analysis and Plans,” AIAA SPACE Conference \& Exposition 2008.

${ }^{7}$ Johnson, M. Hannan, M. Brandon, J. Derry, S. "Ares I-X Flight Test Validation of Control Design Tools in the Frequency Domain,”. JANNAF-1829, 58th JANNAF Propulsion Meeting, April 2011.

${ }^{8}$ Wie, B. Space Vehicle Dynamics and Control. $5^{\text {th }}$ edition, AIAA, Reston VA,1998.

${ }^{9}$ MATLAB/Simulink, Software Package, Ver. 2011b. MathWorks Inc., Natick, Massachusetts. MATLAB and Simulink are registered trademarks of the MathWorks, Inc.

${ }^{10}$ Newsom, J. Mukhopadhyay. V. "Application of Matrix Singular Value Properties for Evaluating Gain and Phase Margins of Multiloop Systems,”. NASA Technical Memorandum. July 1982.

${ }^{11}$ Stevens, B., and Lewis, F., Aircraft Control and Simulation. $2^{\text {nd }}$ ed., Wiley, John \& Sons, Incorporated, New York, 2003.

${ }^{12}$ Blight, J.D., Dailey, R.L., and Gangsaas D. Practical control law design for aircraft using multivariable techniques. Advances in Aircraft Flight Control. Copyright. Mark B. Tischler. 1996. 